\title{
STIMULATED EMISSION AS A MAJOR CHANNEL FOR THE DEPOPULATION OF LASER-EXCITED STATES IN CONDENSED MEDIA \&ٔ
}

\author{
V.A. APKARIAN \\ Department of Chemistry, University of California, Irvine, California 92717, USA
}

Received 5 March 1984; in final form 11 April 1984

\begin{abstract}
In condensed media, due to the high molecular densities, stimulated emission may become the dominant channel for depletion of population in laser-excited states. The phenomenon is fairly general. The criteria necessary for such a process, the specific case of vibrational excitation in matrix-isolated $\mathrm{CH}_{3} \mathrm{~F}$, and some practical implications are considered.
\end{abstract}

\section{Introduction}

Many modem kinetic measurements rely on initial preparation of molecular states by laser excitation. In these experiments it is imperative to know the exact state preparation by the laser pulse, since this is assumed as the initial condition for the kinetic evolution of the system. Analysis of the kinetic equations for radiative energy transfer indicates that in condensed media, even for relatively mild pump fluences, the initially excited state may equilibrate with others via stimulated emission. Thus, the assumption that the pump pulse serves to populate only the directly excired state may be false and hence the interpretation of the slibsequent kinetics, erroneous. These considerations are particularly important in condensed phase studies, where high molecular densities can be achieved without necessarily introducing very efficient non-radiative decay channels. An obvious implication is that if such a system is enclosed within a resonator cavily laser action may ensue.

We consider a very general case in which a high lying state, state 2 of fig. 1 , is directly excited by a laser pulse. State 1 is a state below state 2 that has been singled out from the rest of the lower manifold either. because it is most strongly dipole-coupled to

In Chem. Phys. Letters 108 (1984) 150 the original version of this paper has crroneously been published instead of the revised ont which is reproduced here.

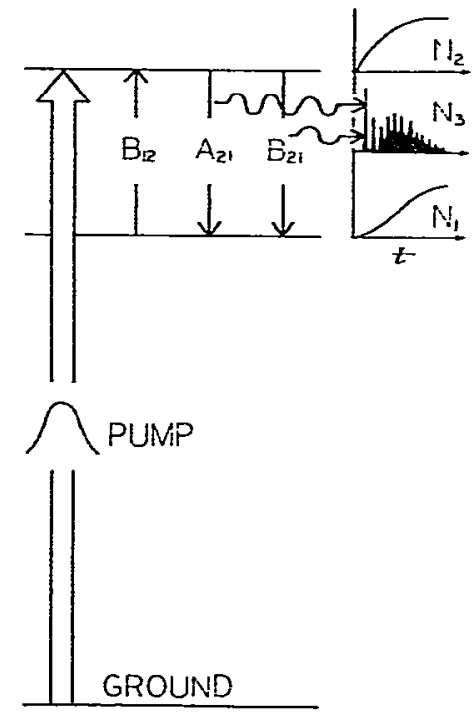

Fig. 1. A schematic representation of the kinetic system under consideration. State 2 is excited by a pump pulse and equilibrates with state 1 through radiative processes alone. The population evolutions in state 1 and $2, N_{1}$ and $N_{2}$, are calculated together with the population of the resident ficld, $N_{3}$ as indicated in the upper-right inset.

state 2, or, in the absence of such a consideration, because it is the nearest, lower, dipole-coupled state. In the absence of efficient non-radiative decay mechanisms, state 2 and 1 will equilibrate according to 
Einstein's rate equations:

$\mathrm{d} N_{2} / \mathrm{d} t=\left(N_{1}-N_{2}\right) B_{12} \rho-N_{2} A_{21}$.

$\mathrm{d} N_{1} / \mathrm{d} t=\left(N_{2}-N_{1}\right) B_{12} \rho+N_{2} A_{21}$,

in which $N_{2}$ and $N_{1}$ are the fractional populations of states 2 and 1 respectively; $B_{12}$ and $A_{21}$ are the usual coefficients of stimulated absorption and spontaneous emission respectively; and $\rho$ is the photon field density in units of erg $\mathrm{cm}^{-3} \mathrm{~Hz}^{-1}$.

In the absence of external radiation sources, $\rho$ is simply duc to photons emitted during $2 \rightarrow 1$ radiative transitions. Thus, we are only considering the processes of self-stimulated emission and self-trapping. We note that of the emitted photons. only those that remain within the volume confining the molecules. are effective in stimulating resonant absorption or emission. Thus $\rho$ represents the resident photon field density created by spontaneous and stimulated emission and degraded by stimulated absorption and more importantly by leakage out of the confinement volume. If the walls of the medium are assumed to be non-reflective. then the leakage rate constant, $k_{1}$, is given by

$k_{1}=c / n l$,

in which $c$ is the speed of light in vacuum: $n$ is the index of refraction of the medium and $l$ is the average distance between any molecule and the walls of the confinement volume. $l$ can easily be derived for a given geometry of the medium (vide infra). A rate equation can now be defined for the fractional population of the resident photon field, $N_{3}$, as

$\mathrm{d} N_{3} / \mathrm{d} t=\left(N_{2}-N_{1}\right) B_{12} \rho+A_{21} N_{2}-k_{1} N_{3}$

and $\rho$ can be identified as

$\rho=(h \nu / \Delta \nu) N N_{3}$,

in which $y$ and $\Delta \nu$ correspond to the frequency and linewidth of the $2 \rightarrow 1$ transition; $N$ is the molecular number density; and $N_{3}$ is the fractional population of the resident photon field such that $N_{3}$ is the photon number density.

The complete kinetics of the system of fig. 1 , can be represented by three coupled rate equations:

$$
\begin{gathered}
\mathrm{d} N_{2} / \mathrm{d} t=k\left(N_{1}-N_{2}\right) N_{3}-A_{21} N_{2} \\
\quad+(2 \delta / \sigma \pi) \exp \left\{-\left[(t-\sigma) / \frac{1}{2} \sigma\right]^{2}\right\},
\end{gathered}
$$

$\mathrm{d} N_{1} / \mathrm{d} t=k\left(N_{2}-N_{1}\right) N_{3}+A_{21} N_{2}$.

$\mathrm{d} N_{3} / \mathrm{d} t=k\left(N_{2}-N_{1}\right) N_{3}+A_{21} N_{2}-k_{1} N_{3}$,

where

$k=B_{12} N / \nu \nu / \Delta \nu=A_{21} c^{3} N / 8 \pi \nu^{2} \Delta \nu$.

The pump pulse is explicitly included by the last term in eq. (5a), as a Gaussian of pulsewidth $\sigma$ seconds, capable of transferring $\delta$ fractional population from the ground state to state 2 .

These coupled differential equations are nonlinear, hence an exact solution can only be obtained by numerical methods. Special methods are required due to the stiffness of the system - in the example to be considered below, the rate constants vary over fifteen orders of magnitude. It is therefore useful to establish criteria that justify the full non-linear treatment. Clearly, under very mild excitation conditions. it should be possible to ignore the contributions of self-induced stimulated processes. In this limit, the rate equations can be linearized and solved analytically. The criterion for the validity of this approximation is

$B_{12} \rho\left(\equiv k N_{3}\right) \ll A_{21}$.

A minimal test for the satisfaction of this condition can be performed by the regressive me thod of linearizing the rate equations with the assumption that eq. (6) holds, solving for $N_{3}$ and checking the assumption by evaluating eq. (6). The criterion of eq. (6) implies that, the resident photon field is created by spontaneously emitted photons only. Furthermore (since there are no competitive channels of population decay), in this linit, the effect of the laser excitation can be regarded as a step rise in the population of state 2. Thus, eqs. (5a) and $(5 c)$ reduce to

$\mathrm{d}_{2} / \mathrm{d} t=-N_{2} A_{21}$.

$\mathrm{d} N_{3} / \mathrm{d} t=N_{2} A_{21}-k_{1} N_{3}$,

which can be solved simultaneously and the resident photon field population can be obtained as

$N_{3}=\left[\delta A_{21} /\left(k_{1}-A_{21}\right)\right]\left[\exp \left(-A_{21} t\right)-\exp \left(-k_{1} t\right)\right]$.

In general $k_{1}(\equiv c / n I) \gg A_{21}$, therefore at early time: 
$N_{3}=\delta A_{21} / k_{1}$.

Using eqs. (9) and (5d), the condition of eq. (6) can be recast in a more useful form:

$A_{21} c^{2} N \delta n l / 8 \pi \nu^{2} \Delta \nu \ll 1$.

The condition of eq. (10) has ro be met in order to justify neglecting the self-stimulated processes in a given system. The derivation has so far been completely general. In order to give numerical examples, we consider a specific case.

\section{Vibrational excitation in matrix-isolated species}

Numerous studies of vibrational energy transfer in matrix-isolated species have been performed $[1,2]$. In these studies. a vibrational level in either the ground or excited electronic state is initially excited by a laser pulse, and the subsequent time evolution of vibrational populations is monitored by fluorescence detection or by double resonance techniques. In the case of vibronic excitation, stimulated emission has already been observed and reported for $\mathrm{C}_{2}$ isolated in Ar matrices [3]. In these experiments vibrational levels in the $\mathrm{d}^{3} \Pi_{\mathrm{g}}$ electronic manifold were excited by two photon absorption and stimulated emission was observed on numerous lines of the Swan band $\left(d^{3} \Pi_{g} \rightarrow\right.$ a $3 \Pi_{u}$ ). For the purposes of lifetime measurement, stimulated emission had to be eliminated by the insertion of neutral density filters in the pump beam [3].

In the case of vibrational excitation in the ground electronic state, it is well known that at cryogenic temperatures, when the lowest fundamental is initially excited, population inversion and hence stimulated enission in the overtones can be achieved by the Treanor effect [4]. The overtones in this case are populated by the ladder-climbing mechanism of long-range, phonon assisted $V-V$ transfer. The population inversion is due to anharmonicity shifts and the discrepancy between vibrational and bath temperatures [4] and can be conveniently predicted by calculating the vibrational temperatures of the system [5]. These predictions were most recently verified by demonstrating laser action in the overtones of $\mathrm{CN}^{-}$doped in an ionic crystal, held at cryogenic temperatures [6].

Here we consider a very different situation; namely, the outcome of directly exciting a state other than the lowest fundamental. As a typical example, we first consider the outcome of direct excitation of $2 \nu_{3}$ in matrix isolated $\mathrm{CH}_{3} \mathrm{~F}$. This situation is similar to the experimental system of Young and Moore, where the $3 \mu$ states of $\mathrm{CH}_{3} \mathrm{~F}$ were excited in rare gas matrices [7]. The laser parameters to be used are derived from those studies. It should be noted that $2 \nu_{3}$ is not directly excited in these experiments, however population from the $3 \mu$ states is transferred directly to $2 \nu_{3}$ within the excitation pulse width (at a rate $<5 \mathrm{~ns}$ ), via intramolecular energy transfer [7]. It is also worth noting that in these investigations, the effect of stimulated absorption was taken into account, however, stimulated emission was not considered.

The pertinent parameters for the evaluation of the criterion of eq. (10) are: $A_{2 \nu_{3} \nu_{3}}=30 \mathrm{~s}^{-1 \neq} ; \nu / c \approx$ $1000 \mathrm{~cm}^{-1} ; \Delta \nu \approx 3 \times 10^{9}[8] ; N=1 \times 10^{19} \mathrm{~cm}^{-3}$ (assuming a dilution ratio of $1: 1000$ ); $n \approx 1.3[9] ; \delta$ $=0.01$ (assumes that $1 \%$ of the population is excited by the laser); for a $1 \mathrm{~mm}$ thick, non-scattering matrix, $l=0.075 \mathrm{~cm}^{\neq}$. Using these values, the lhs of eq. (10) can be evaluated as $\approx 4$. Thus, the condition for ignoring the stimulated rate processes is not met, and an exact evaluation of the non-linear rate equations, eq. (5), is necessary.

The coupled rate equations, eq. (5), were evaluated by numerical integration. The time evolutions of the populations in states 2,1 and the resident field are illustrated in fig. 2 for the duration of the pump pulse. In all cases a pump pulse of $10 \mathrm{~ns}$ full-width, $\delta=0.01$ and $k_{1}=10^{11} \mathrm{~s}^{-1}$ were used. $k$ was varied about $10^{14} \mathrm{~s}^{-1}$, the value obtained by the above system parameters. Complete equilibration between the two states would imply equal populations, i.e. $N_{2}=N_{1}=$ $\frac{1}{2} \delta$. This limit is indicated in each plot. For values

$A_{2 \nu_{3}, \nu_{3}}$ is assumed to be twice $A_{v_{3}, 0}$. The radiative lifetime of $\nu_{3}$ and its linewidth in Kr matrices have been measured, see ref. [8].

₹ For a non-scattering matrix, ignoring the possibility of total internal reflection, $I$ is simply the average distance from any trapped molecule to the matrix boundaries. The usual geometry of matrices is that of a disk, assuming an isotropic distribution of molecules within the disk, it can be shown by symmetry arguments that the average distance from any molecule to the walls is the same as the average distance from the center of the matrix to the walls. Thus, $l$ is the ratio of the sum of all distances to the number of all distances from the center to the walls, i.e. $l=3 V / 4 \pi r^{2}$ and for a disk, $l=3 d / 4$, where $d$ is the thickness of the disk. 

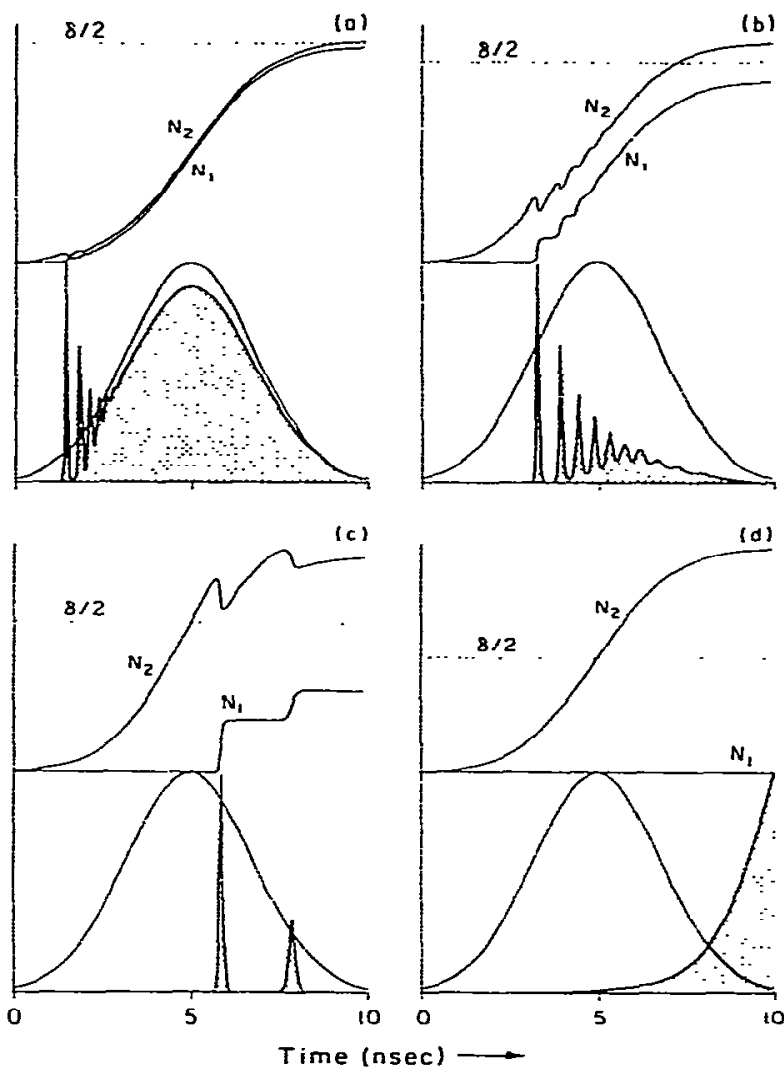

Fig. 2. The numerical solution of the coupled non-linear equations, eq. (5), is illustrated. $k_{1}=10^{11} \mathrm{~s}^{-1}: A_{21}=30$ $\mathrm{s}^{-1}$ is used in all cases while $k$ is varied: (a) $k=10^{15} \mathrm{~s}^{-1}$, (b) $k=10^{14} \mathrm{~s}^{-1}$. (c) $k=2 \times 10^{13} \mathrm{~s}^{-1}$. (d) $k=10^{13} \mathrm{~s}^{-1}$. In each case the populations of states 2 and 1 are presented, normalized to the state 2 population, to facilitate interpretation, the complete equilibration limit of $N_{1}=N_{2}=\frac{1}{2} \delta$ is indicated by the dotted line. In the lower panels the time cvolutions of the pump pulse and the resident field (shacied area) are plotted, in arbitrary units. The time axis in all cases extends to $10 \mathrm{~ns}$, the full width of the pump pulse.

of $k>10^{13}$, avalanches triggered by spontaneously emitted photons equilibrate the two states. The equilibration is complete for $k=10^{15}$ (fig. 2a). A sharp threshold exists for this process. While no avalanches can be sustained at $k=10^{13}$ (fig. 2d), at $k=2 \times 10^{13}$, in two avalanches, $50 \%$ equilibration is achieved (fig. $2 c$ ). The observed oscillations in figs. $2 a-2 c$ are uue strictly to the non-linear, autocatalytic (positive feedback) nature of stimulated emission. The upswings in the oscillations correspond to avalanching where stim- ulated photons further stimulate the emission of photons. The downswings start when stimulated emission is quenched by the instantaneous equilibration of populations in the upper and lower states, $N_{1}(t)=N_{2}(t)$. In order to sustain these oscillations, the resident field should be able to grow. The condition for growth in the resident field can be derived from eq. (5c) as: $\left(N_{2}-N_{1}\right) k$ $>k_{1}$. As long as state 1 does not possess a significant ambient population, $\delta k>k_{1}$ can be taken as the threshold condition. Note that this is the condition for avalanching; even when this condition is not met, a significant population may be transferred via stimulated emission during the lifetime of state 2 . The criterion of eq. (10) has to be met in order to avoid this situation. While this effect is less dramatic, it should be taken into account in accurate lifetime measurements.

The critical rate constants of the generalized system are $k$ and $k_{1}$. The competition between their associated rate processes determines the efficiency of radiative equilibration. $A_{21}$ mainly serves as a trigger for the avalanches. The explicit dependence of $k$, the rate constant for stimulated processes, was presented in eq. (5d). It is directly proportional to the molecular density, $N$, hence its importance in condensed media. Furthermore $k$ has an overall linear dependence on frequency of the $2 \rightarrow 1$ transition (an explicit $\nu^{-2}$ dependence and an implicit $\nu^{3}$ dependence through its proportionality to $A_{21}$, see eq. (5d)); hence the importance of the process in vibronic transitions. The leakage rate, $k_{l}$, is defined by the geometry of the medium and the reflectivity of its walls. If the system were enclosed in completely reflective walls, the leakage rate would reduce to zero. If the system were enclosed in a two-mirror resonator cavity, $k_{1}$ would be reduced according to $k_{1}=(c / n l)\left[1-\ln \left(R_{1} R_{2}\right)\right]$, where now $l$ is the length of the medium along the axis of the cavity, and $R_{1}$ and $R_{2}$ are the reflectivities of the cavity mirrors [10]. As long as the avalanching condition is met with this definition of $k_{1}$, laser action should be possible. While cryogenic matrices may not be practical for this purpose, room temperature matrices, such as doped ionic crystals are attractive candidates.

\section{Conclusions}

It should be clear from the above development that, under the proper conditions, these systems could be 
made to lase. However, it is more important to note that, stimulated emission may proceed under the mild excitation conditions used in kinetic studies and should therefore be taken into account as a possible channel for energy transfer. The situation is not limited to the examples chosen above. The process is more general and could be applied to many experimental conditions; such as vibronic or rovibrational excitation, excitation in liquids as well as solids and clearly the systems need not be held at low temperatures. Perhaps the most important assumption in the general model is that of the absence of rate processes that may compete with the considered radiative channels. These rate processes should therefore be considered as possible channels of encrgy transfer and their neglect should be based on rigorous criteria, such as the ones presented above.

\section{References}

[1] F. Lcgay, in: Chemical and biochemical applications of lasers, Vol. 2, ed. C.B. Moore (Academic Press, New York, 1977).

[2] V.E. Bondybey and L.E. Brus, Advan. Chem. Phys. 41 (1980) 269.

[3] V.E. Bondybey, J. Chem. Phys. 65 (1976) 2296.

[4] C.E. Treanor, J.W. Rich and R.G. Rchm, J. Chem. Plyys. 48 (1968) 1798.

[5] V.A. Apkarian and E.Weitz, Chem. Phys. Letters 76 (1980) 68.

[6] R.W. Tkach, T.R. Gosnell and A.J. Sievers, Opt. Letters 10 (1984) 12 ?

[7] L. Yound and C.B. Moore, J. Chem. Phys. 76 (1982) 5869.

[8] V.A. Apkarian and E.Weitz, J. Chem. Phys. 76 (1982) 5796.

[9] A.C. Sinnock and B.L. Smith, Phy's. Rev. 181 (1969) 1297.

[10] A. Yaziv, Quantum clectronics (Wiley, New York, 1975) ch. 7. 